\title{
Genus of The Hypercube Graph And Real Moment-Angle Complexes
}

\author{
Shouman Das* \\ Department of Mathematics, University of Rochester, Rochester NY USA
}

\begin{abstract}
In this paper we demonstrate a calculation to find the genus of the hypercube graph $Q_{n}$ using real moment-angle complex $\mathcal{Z}_{\mathcal{K}}\left(D^{1}, S^{0}\right)$ where $\mathcal{K}$ is the boundary of an $n$-gon. We also calculate an upper bound for the genus of the quotient graph $Q_{n} / C_{n}$, where $C_{n}$ represents the cyclic group with $n$ elements.

Keywords: Topology, polyhedral product, moment-angle complex, graph theory, combinatorics

2010 MSC: $05 \mathrm{C} 10,57 \mathrm{M} 15$
\end{abstract}

\section{Contents}

1 Genus of a Graph 2

2 Moment-Angle Complex 5

3 Action of $C_{n}$ on $Q_{n}$ and $\mathcal{Z}_{\mathcal{K}_{n}}\left(D^{1}, S^{0}\right)$

3.1 Branched covering and genus of $\mathcal{Z}_{\mathcal{K}}\left(D^{1}, S^{0}\right) / C_{n} \ldots \ldots$

3.2 An upper bound for genus of quotient graph $Q_{n} / C_{n} \ldots 14$

\section{Introduction}

In graph theory, the hypercube graph is defined as the 1-skeleton of the $n$-dimensional cube. The graph theoretical properties of this graph has been studied extensively by Harary et al in [1]. It is well known that this graph

\footnotetext{
*Corresponding author

Email address: shouman.das@rochester.edu (Shouman Das)
} 
has genus $1+(n-4) 2^{n-3}$. This fact was proved by Ringel in [2], Beineke and Harary in [3]. The moment-angle complex or polyhedral product has been studied recently in the works of Buchstaber and Panov [4], Denham and Suciu [5], Bahri et al. [6]. In this paper, we give an embedding of the hypercube graph in the real moment-angle complex and calculate the genus of the hypercube graph. This demonstrates an interesting relationship between the geometry of hypercube graph and real moment-angle complex.

\section{Genus of a Graph}

Definition 1.1. The hypercube graph $Q_{n}$ for $n \geq 1$ is defined with the following vertex and edge sets.

$$
\begin{aligned}
V & =\left\{\left(a_{1}, \cdots, a_{n}\right) \mid a_{i}=0 \text { or } 1\right\} \\
& =\text { the set of all ordered binary } n \text {-tuples with entries of } 0 \text { and } 1 \\
E & =\{(u, v) \in V \times V \mid u \text { and } v \text { differ at exactly one place }\}
\end{aligned}
$$

It is straightforward to see that the hypercube graph can also be defined recursively as a cartesian product [7, p. 22].

$$
Q_{1}=K_{2}, \quad Q_{n}=K_{2} \square Q_{n-1}
$$

Now we will define the genus of a graph. In this paper, a 'surface' will mean a closed compact orientable manifold of dimension of 2. A graph embedding in a surface means a continuous one to one mapping from the topological representation of the graph into the surface. More explanation about graph embeddings can be found at [8].

Definition 1.2. The genus $\gamma(G)$ of a graph $G$ is the minimal integer $n$ such that the graph can be embedded in the surface of genus $n$. In other words, it is the minimum number of handles which needs to be added to the 2-sphere such that the graph can be embedded in the surface without any edges crossing each other.

Example 1.3. All planar graphs have genus 0. The complete graph with 5 vertices denoted by $K_{5}$ and the complete bipartite graph with 6 vertices denoted by $K_{3,3}$ both have genus 1 ( Figure 1.2). The non-planarity of these graphs denoted by $K_{n}$ and $K_{m, n}$ are explained in [9, chapter 6]. 

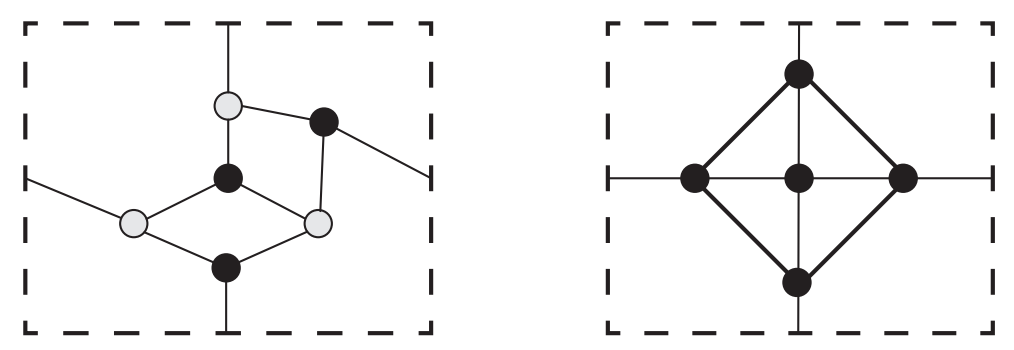

Figure 1: $K_{3,3}$ and $K_{5}$ embedded in a torus.

Definition 1.4 (2-cell embedding). Assume that $\mathrm{G}$ is a graph embedded in a surface. Each region of the complement of the graph is called a face. If each face is homeomorphic to an open disk, this embedding is called a 2-cell embedding.

In this paper we will restrict our attention to 2-cell embeddings of graphs because the embedding of the hypercube graph in a real moment-angle complex is a 2-cell embedding. We describe it in the next section.

Now restricting our attention to 2-cell embeddings of a graph $G$ in a surface with genus $g$, we can see that

$$
\gamma_{M}(G)=\max \{g \mid G \text { has a 2-cell embedding on a surface with genus } g\}
$$

must exist. This is true because if a graph has a 2-cell embedding in a surface $S_{g}$, then each handle of the surface must contain at least one edge. So we have a loose upper bound of $\gamma_{M}(G) \leq e$ (See [10] for further explanation). So we can define the maximum genus of a finite connected graph as follows.

Definition 1.5 (Maximum genus). The maximum genus $\gamma_{M}(G)$ of a connected finite graph $G$ is the maximal integer $m$ such that $G$ has a 2-cell embedding on the surface of genus $m$.

Two theorems which are important tools in the analysis of graph embeddings follow next.

Theorem 1.6 (Euler's Formula). Let a graph $G$ has a 2-cell embedding in the surface $S_{g}$ of genus $g$, with the usual parameter $V, E, F$. Then

$$
|V|-|E|+|F|=2-2 g
$$

Proof. See [8, Chapter 3] 
Theorem 1.7 (Duke[11]). A graph $G$ has a 2-cell embedding in a surface $S_{g}$ of genus $g$ if and only if $\gamma(G) \leq g \leq \gamma_{M}(G)$.

The last theorem tells us that if there exist 2-cell embeddings of a graph in surfaces of genera $m$ and $n$ with $m \leq n$, then for any integer $k$ with $m \leq k \leq n$, there exists a 2-cell embedding of the graph in a surface with genus $k$. A detailed explanation and proof of this theorem can be found in Richard A. Duke's original paper [11].

Using these theorems, we can find a lower bound for the genus of the hypercube graph. Let a graph $G$ is embedded in a surface and $f_{i}$ denote the number of faces which has $i$ edges as its boundary. So we have

$$
2|E|=\sum_{i} i f_{i}
$$

For the hypercube graph, each face must have at least 4 edges as its boundary. Therefore,

$$
2|E|=\sum_{i \geq 4} i f_{i} \geq \sum_{i} 4 f_{i}=4|F|
$$

which implies $|F| \leq \frac{|E|}{2}$. Now using Euler's formula,

$$
\begin{aligned}
g= & 1-\frac{|V|}{2}+\frac{|E|}{2}-\frac{|F|}{2} \\
& \geq 1-\frac{|V|}{2}+\frac{|E|}{2}-\frac{|E|}{4}=1-\frac{|V|}{2}+\frac{|E|}{4}
\end{aligned}
$$

But for the hypercube graph $Q_{n}$, we have $|V|=2^{n},|E|=n 2^{n-1}$. So using the above inequality we get a lower bound 1 for the genus of a hypercube graph,

$$
\gamma\left(Q_{n}\right) \geq 1-2^{n-1}+n 2^{n-3}=1+(n-4) 2^{n-3}
$$

To show that this lower bound can be achieved, we will use the real moment-angle complex. In fact, we prove the following theorem.

Theorem 1.8. For $n \geq 3$, the hypercube graph can be embedded in a surface with genus $1+(n-4) 2^{n-3}$. Moreover, this embedding is a 2-cell embedding.

\footnotetext{
${ }^{1}$ See [12] for more detail on the inequalities involving genus of a graph
} 


\section{Moment-Angle Complex}

Definition 2.1. Let $(X, A)$ be a pair of topological spaces and $\mathcal{K}$ be a finite simplicial complex on a set $[m]=\{1, \cdots, m\}$. For each face $\sigma \in \mathcal{K}$, define

$$
(X, A)^{\sigma}=Y_{1} \times \cdots \times Y_{m}
$$

where

$$
Y_{i}=\left\{\begin{array}{lll}
X & \text { if } & i \in \sigma \\
A & \text { if } & i \notin \sigma
\end{array}\right.
$$

The moment-angle complex $\mathcal{Z}_{\mathcal{K}}(X, A)$ corresponding to pair $(X, A)$ and simplicial complex $\mathcal{K}$ is defines as the following subspace of the cartesian product $X^{m}$.

$$
\mathcal{Z}_{\mathcal{K}}(X, A)=\bigcup_{\sigma \in \mathcal{K}}(X, A)^{\sigma}
$$

For our calculation we will use the pair $\left(D^{1}, S^{0}\right)$. This space, $\mathcal{Z}_{\mathcal{K}}\left(D^{1}, S^{0}\right)$ is called the real moment-angle complex corresponding to $\mathcal{K}$.

Example 2.2. Let $\mathcal{L}_{n}$ denote the simplicial complex with $n$ discrete points. Then by the above definition

$$
\begin{gathered}
\mathcal{Z}_{\mathcal{L}_{n}}\left(D^{1}, S^{0}\right)=\left(D^{1} \times S^{0} \times \cdots \times S^{0}\right) \cup\left(S^{0} \times D^{1} \times\right. \\
\left.\cdots \times S^{0}\right) \cup \cdots \cup\left(S^{0} \times S^{0} \times \cdots \times D^{1}\right)
\end{gathered}
$$

It is easy to see that $\mathcal{Z}_{\mathcal{L}_{n}}\left(D^{1}, S^{0}\right)$ is homeomorphic to of the hypercube graph $Q_{n}$.

From the definition of the moment-angle complex, we can prove the following lemma.

Lemma 2.3. Let $f: \mathcal{L} \hookrightarrow \mathcal{K}$ be an inclusion map of simplicial complex where $\mathcal{L}$ and $\mathcal{K}$ both have the same number of vertices. Then there exists an inclusion map of moment-angle complexes, $\mathcal{Z}_{f}: \mathcal{Z}_{\mathcal{L}}(X, A) \hookrightarrow \mathcal{Z}_{\mathcal{K}}(X, A)$.

Proof. We can consider $\mathcal{L}$ as a subcomplex of $\mathcal{K}$. So any face $\sigma$ of $\mathcal{L}$ is also a face of $\mathcal{K}$. From this we can conclude that

$$
(X, A)^{\sigma} \subset \bigcup_{\tau \in \mathcal{K}}(X, A)^{\tau}
$$

This implies that $\mathcal{Z}_{\mathcal{L}}(X, A) \subset \mathcal{Z}_{K}(X, A)$. 
Example 2.4. Let $\mathcal{K}_{n}$ be the boundary of an $n$-gon and $\mathcal{L}_{n}$ be the $n$ vertices of $\mathcal{K}_{n}$. Using the above lemma, we can conclude that $\mathcal{Z}_{\mathcal{L}_{n}}\left(D^{1}, S^{0}\right)=$ $Q_{n}$ is embedded in $\mathcal{Z}_{K_{n}}\left(D^{1}, S^{0}\right)$. Also, if we consider the complement of $\mathcal{Z}_{\mathcal{L}_{n}}\left(D^{1}, S^{0}\right)$ in $\mathcal{Z}_{K_{n}}\left(D^{1}, S^{0}\right)$, we will get a collection of open discs $\left(D^{1} \times D^{1}\right)^{\mathrm{o}}$ which is straightforward from the definitions. So, this embedding of $Q_{n}$ in $\mathcal{Z}_{K_{n}}\left(D^{1}, S^{0}\right)$ is clearly a 2 -cell embedding.

It is interesting to note that $\mathcal{Z}_{K_{n}}\left(D^{1}, S^{0}\right)$ is a closed compact surface with genus $1+(n-4) 2^{n-3}$. This fact was proved by Coxeter in [13]. We will give an inductive proof here.

Proposition 2.5. For $n \geq 3, \mathcal{Z}_{K_{n}}\left(D^{1}, S^{0}\right)$ is a closed, compact and orientable surface with genus $1+(n-4) 2^{n-3}$.

Proof. For brevity, we write $\mathcal{Z}_{\mathcal{K}_{n}}$ to denote $\mathcal{Z}_{\mathcal{K}_{n}}\left(D^{1}, S^{0}\right)$.

If $n=3$, it is straightforward that $\mathcal{Z}_{\mathcal{K}_{n}}=\partial\left(D^{1} \times D^{1} \times D^{1}\right) \approx S^{2}$. Let's assume the statement is true for an integer $n \geq 3$. So $\mathcal{Z}_{\mathcal{K}_{n}}$ is an orientable surface of genus $1+(n-4) 2^{n-3}$. Also note that

$$
\begin{aligned}
\mathcal{Z}_{\mathcal{K}_{n}}= & \overbrace{D^{1} \times D^{1} \times S^{0} \times \cdots \cdots \times S^{0}}^{n \text { factors }} \\
& \cup S^{0} \times D^{1} \times D^{1} \times S^{0} \times \cdots \times S^{0} \\
& \vdots \\
& \cup S^{0} \times \cdots \cdots \cdots \times S^{0} \times D^{1} \times D^{1} \\
& \cup D^{1} \times S^{0} \cdots \cdots \cdots \cdots \times S^{0} \times D^{1}
\end{aligned}
$$

Let $B$ be the last term in the union that is $B=D^{1} \times S^{0} \times \cdots \times S^{0} \times D^{1} \subset$ $\mathcal{Z}_{\mathcal{K}_{n}}$. So $B$ is $2^{n-2}$ copies of $D^{1} \times D^{1}$ on the surface $\mathcal{Z}_{\mathcal{K}_{n}}$. Now note that,

$$
\partial(B)=\left(S^{0} \times S^{0} \times \cdots \times S^{0} \times D^{1}\right) \cup\left(D^{1} \times S^{0} \times \cdots \times S^{0} \times S^{0}\right)
$$

and

$$
\mathcal{Z}_{\mathcal{K}_{n+1}}=\left(\left(\mathcal{Z}_{\mathcal{K}_{n}}-B\right) \times S^{0}\right) \cup\left(\partial B \times D^{1}\right)
$$

This means that to construct $\mathcal{Z}_{\mathcal{K}_{n+1}}$, we first delete $2^{n-2}$ copies of $D^{1} \times D^{1}$ from $\mathcal{Z}_{\mathcal{K}_{n}}$, then take two copies of $\mathcal{Z}_{\mathcal{K}_{n}}-B$ and glue $2^{n-2}$ copies of 1 -handle along the boundary of $B$. Therefore,

$$
\mathcal{Z}_{\mathcal{K}_{n+1}}=\mathcal{Z}_{\mathcal{K}_{n}} \# \mathcal{Z}_{\mathcal{K}_{n}} \#\left(2^{n-2}-1\right) S^{1} \times S^{1}
$$


Here one of the $2^{n-2}$ handles is being used to construct the first connected sum $\mathcal{Z}_{\mathcal{K}_{n}} \# \mathcal{Z}_{\mathcal{K}_{n}}$ and the remaining $2^{n-2}-1$ copies of 1 -handles are connected as $2^{n-2}-1$ copies of torus. So clearly $\mathcal{Z}_{\mathcal{K}_{n+1}}$ is a closed compact orientable surface with genus

$$
2\left(1+(n-4) 2^{n-3}\right)+2^{n-2}-1=1+((n+1)-4) 2^{(n+1)-3} .
$$

In the above discussion, we have proved that the hypercube graph $Q_{n}$ can be embedded in the real moment-angle complex $\mathcal{Z}_{\mathcal{K}_{n}}\left(D^{1}, S^{0}\right)$ which is a surface of genus $1+(n-4) 2^{n-3}$. Hence Theorem 1.8 is proved.

\section{Action of $C_{n}$ on $Q_{n}$ and $\mathcal{Z}_{\mathcal{K}_{n}}\left(D^{1}, S^{0}\right)$}

Let $C_{n}$ denote the cyclic group with $n$ elements generated by $\sigma$. Since $\mathcal{K}_{n}$ can be considered as the boundary of a regular $n$-gon, we can define an action of $C_{n}$ on $\mathcal{K}_{n}$ by rotating the $n$-gon by $2 \pi / n$ radians about the centre. If $(i, i+1)$ represents an edge, then this action will take this edge to $(i+1, i+2)$ (here the vertices are considered as $i(\bmod n))$. So We can define an action of $C_{n}$ on $\mathcal{Z}_{\mathcal{K}_{n}}\left(D^{1}, S^{0}\right)$ by $\sigma\left(x_{1}, \cdots, x_{n}\right)=\left(x_{\sigma(1)}, \cdots, x_{\sigma(n)}\right)=\left(x_{2}, \cdots, x_{n}, x_{1}\right)$ where $\left(x_{1}, \cdots, x_{n}\right) \in\left(D^{1}, S^{0}\right)^{\tau}$ for a maximal face $\tau \in \mathcal{K}_{n}$. So $\sigma$ is rotating the coordinates of a point in the moment-angle complex $\mathcal{Z}_{\mathcal{K}_{n}}\left(D^{1}, S^{0}\right)$. We can define a similar action of $C_{n}$ on the hypercube graph $Q_{n}$ by rotating the coordinates of a point. It is straightforward to note that the following diagram commutes.

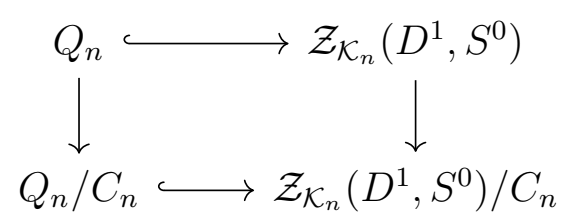

Therefore the quotient graph $Q_{n} / C_{n}$ is embedded in the quotient space $\mathcal{Z}_{\mathcal{K}_{n}}\left(D^{1}, S^{0}\right) / C_{n}$. We will show that the quotient space $\mathcal{Z}_{\mathcal{K}_{n}}\left(D^{1}, S^{0}\right) / C_{n}$ is also a closed connected orientable surface. Therefore, calculating the genus of the surface $\mathcal{Z}_{\mathcal{K}_{n}}\left(D^{1}, S^{0}\right) / C_{n}$ would suffice to give an upper bound for the genus of the quotient graph $Q_{n} / C_{n}$. First we will prove that $\mathcal{Z}_{\mathcal{K}_{n}} / C_{n}$ is closed connected, compact and orientable manifold. Then we will calculate the genus of this surface. Indeed the following theorem can be found in Ali's thesis [14] theorem 4.2.2. 
Theorem 3.1. Let $C_{m}$ be the subgroup of $C_{n}$ i.e. $m \mid n$, then $\mathcal{Z}_{\mathcal{K}_{n}}\left(D^{1}, S^{0}\right) / C_{m}$ is a closed surface.

It is not surprising that the quotient surface $\mathcal{Z}_{\mathcal{K}}\left(D^{1}, S^{0}\right) / C_{n}$ must be an orientable surface. We can prove it by giving a $\delta$-complex structure on this surface and check that all the triangles on the surface can be given an orientation such that any two neighboring triangles' edges fit nicely.

Lemma 3.2. The surface $\mathcal{Z}_{\mathcal{K}_{n}}\left(D^{1}, S^{0}\right) / C_{n}$ is an orientable surface.

Proof. First note that the action of $C_{n}$ permutes the coordinate of a point in a cyclic manner. So we only need to consider the space $D^{1} \times D^{1} \times S^{0} \times \cdots \times S^{0}$, which is actually $2^{n-2}$ copies of $D^{1} \times D^{1}$. For each of these squares, we draw a diagonal from the lower left corner to the top right, make a triangulation of the surface and give suitable orientation to each triangle. Then we glue these squares along their boundaries under the identification generated by $C_{n}$. Let $\epsilon_{1} \epsilon_{2} \ldots \epsilon_{n}$ represent the coordinate $\left(\epsilon_{1}, \ldots, \epsilon_{n}\right)$ where $\epsilon_{i}=0$ or 1 . For abbreviation, we write directed edges as $(000,010)$ which represent the directed edge from $(0,0,0)$ to $(0,1,0)$.

Case 1: $(n=3)$. We have two copies of $D^{1} \times D^{1}$ (Figure 2a). Under the action of $C_{n}$, we have the following identification of edges on the boundary of this two squares.

$$
\begin{array}{ll}
(000,010) \sim(000,100), & (001,011) \sim(010,110), \\
(100,110) \sim(001,101), \quad(101,111) \sim(011,111)
\end{array}
$$

As shown in the figure 2, we can give orientation to to each of the triangles such that the orientation of each edge fits together.

Case 2: $(n=4)$. In this case, we have four copies of $D^{1} \times D^{1}$ as shown in (Figure $3 \mathrm{~b}$ ). And we have the identification of edges as follows:

$$
\begin{array}{ll}
(0000,0100) \sim(0000,1000), & (0010,0110) \sim(0100,1100), \\
(1000,1100) \sim(0001,1001), & (1011,1111) \sim(0111,1111) \\
(1001,1101) \sim(0011,1011), & (0011,0111) \sim(0110,1110), \\
(1010,1110) \sim(0101,1101), & (0001,0101) \sim(0010,1010)
\end{array}
$$

We can see from the figure that the orientation of each triangle is compatible to each other. 


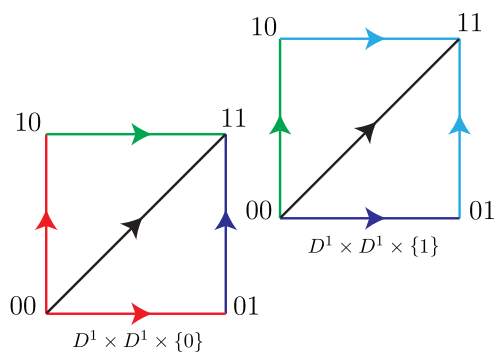

(a)

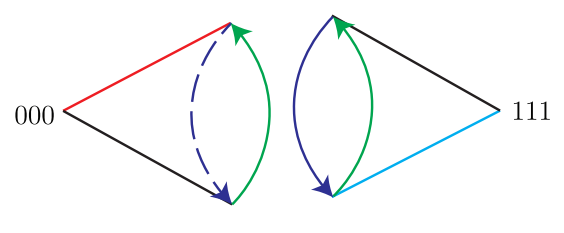

(b)

Figure 2: Quotient space $\mathcal{Z}_{\mathcal{K}_{3}}\left(D^{1}, S^{0}\right) / C_{3}$.
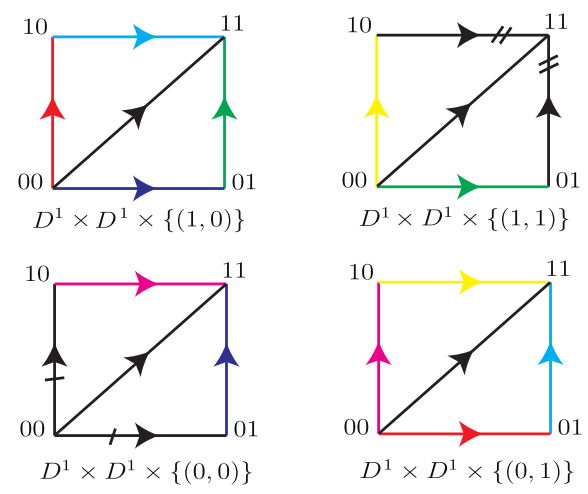

Figure 3: Quotient space $\mathcal{Z}_{\mathcal{K}_{4}}\left(D^{1}, S^{0}\right) / C_{4}$. 
Case 3: $(n \geq 5)$. For $n \geq 5$, we can have the identification of edges as follows:

$$
\begin{array}{lll}
(0000 x, 1000 x) \sim(000 x 0,010 x 0), & (0010 x, 0110 x) \sim(010 x 0,110 x 0), \\
(100 x 0,110 x 0) \sim(00 x 01,10 x 01), & (101 x 1,111 x 1) \sim(01 x 11,1 x 111) \\
(1001 x, 1101 x) \sim(001 x 1,101 x 1), & (001 x 1,011 x 1) \sim(01 x 10,11 x 10), \\
(1010 x, 1110 x) \sim(010 x 1,110 x 1), & (000 x 1,010 x 1) \sim(00 x 10,10 x 10)
\end{array}
$$

Here, $x$ represents a string of length $(n-4)$ whose characters can be 0 or 1. So we can see that all this identification preserve the orientation of the surface. Therefore, $\mathcal{Z}_{\mathcal{K}}\left(D^{1}, S^{0}\right) / C_{n}$ is an orientable surface.

Since the quotient of a compact and connected space is also a compact and connected space, we have proved the following theorem.

Theorem 3.3. Let $\mathcal{K}$ be the boundary of an n-gon. Then $\mathcal{Z}_{\mathcal{K}}\left(D^{1}, S^{0}\right) / C_{n}$ is a closed, compact and orientable surface.

\subsection{Branched covering and genus of $\mathcal{Z}_{\mathcal{K}}\left(D^{1}, S^{0}\right) / C_{n}$}

Next, we will prove the following lemma which gives a formula for finding the genus of the quotient space.

Lemma 3.4. The genus of $\mathcal{Z}_{\mathcal{K}}\left(D^{1}, S^{0}\right) / C_{n}$ is given by the following formula:

$$
g\left(\mathcal{Z}_{\mathcal{K}}\left(D^{1}, S^{0}\right) / C_{n}\right)=1+2^{n-3}-\frac{1}{2 n} \sum_{d \mid n} \phi(d) 2^{n / d}
$$

where $\phi$ is the Euler totient function.

To prove this lemma, we will use the Riemann-Hurwitz formula for branched covering. Note that the quotient map $\mathcal{Z}_{\mathcal{K}}\left(D^{1}, S^{0}\right) \rightarrow \mathcal{Z}_{\mathcal{K}}\left(D^{1}, S^{0}\right) / C_{n}$ would be a covering map if remove a finite number of points (the corners of each $\left.D^{1} \times D^{1}\right)$. So this quotient map is a branched cover. We use the following definition from [15].

Definition 3.5. Let $X$ and $Y$ be two surfaces. A map $p: X \rightarrow Y$ is called a branched covering if there exists a codimension 2 subset $S \subset Y$ such that $p: X \backslash p^{-1}(S) \rightarrow Y \backslash S$ is a covering map. The set $\mathrm{S}$ is called the branch set and the preimage $p^{-1}(S)$ is called the singular set. 
Definition 3.6. Let $p: X \rightarrow Y$ be a branched covering of two surfaces where $Y$ is connected. The degree of this branched covering is the number of sheets of the induced covering after removing the branch points and singular points.

In [14], it is proved that $\mathcal{Z}_{\mathcal{K}}\left(D^{1}, S^{0}\right) \rightarrow \mathcal{Z}_{\mathcal{K}}\left(D^{1}, S^{0}\right) / C_{n}$ is a branched covering of degree $n$. Since the quotient is closed, compact and orientable we can apply the classical Riemann-Hurwitz formula for branched covering.

Theorem 3.7 (Riemann-Hurwitz Formula). Let $G$ be a finite group acting on the surface $X$, such that the map $p: X \rightarrow X / G$ be a branched covering with a branch subset $S \subset X / G$. Let $G_{y}$ represent the isotropy subgroup for a point $y \in X$ and $\chi(X)$ be the Euler characteristic of $X$. Then

$$
\chi(X)=|G| \cdot \chi(X / G)-\sum_{x \in S}\left(|G|-\frac{|G|}{n_{x}}\right)
$$

with $n_{x}=\left|G_{y}\right|$ for $y \in p^{-1}(x)$ and $x \in S$.

To apply this formula we need to calculate the cardinality of the isotropy subgroup for each of the singular points in $\mathcal{Z}_{K}\left(D^{1}, S^{0}\right)$. It is straightforward that the action of $C_{n}$ on a point permute its coordinate in a cyclic manner. The only points in $\mathcal{Z}_{K}\left(D^{1}, S^{0}\right)$ which have a nontrivial isotropy group have coordinates 0 's or 1's only. Therefore, the cardinality of the isotropy subgroup is related to the number of aperiodic necklaces with 2-coloring. We will give some necessary definitions related to aperiodic necklaces and then count the Euler characteristic of $\mathcal{Z}_{K}\left(D^{1}, S^{0}\right) / C_{n}$ by using the Riemann-Hurwitz formula.

Definition 3.8. Let $W$ represent a word of length $n$ over an alphabet of size $k$. We define an action of the cyclic group $C_{n}=\langle\sigma\rangle$ on $\mathrm{W}$ by rotating its characters. For example, if $W=a_{1} a_{2} \cdots a_{n}$ where each $a_{i}$ is a character from the alphabet, then $\sigma(W)=a_{2} a_{3} \cdots a_{n} a_{1}$. A word $W$ of length $n$ is called an aperiodic word if $W$ has $n$ distinct rotation.

Definition 3.9. An equivalence class of an aperiodic word under rotation is called a primitive necklace.

The total number of primitive $n$-necklaces on an alphabet of size k, denoted by $M(k, n)$, is given by Moreau's formula [16],

$$
M(k, n)=\frac{1}{n} \sum_{d \mid n} \mu(d) k^{n / d}
$$


Note that we can deduce Moreau's formula by using Möbius inversion formula and the fact that

$$
k^{n}=\sum_{d \mid n} d M(k, d)
$$

Total Number of Necklace of length $n$ with $k$-coloring: Note that this number is the same as $\sum_{d \mid n} M(k, d)$ since $M(k, d)$ gives us the number of aperiodic necklaces for each divisor $d$ of $n$. So, we have

$$
\begin{aligned}
\sum_{d \mid n} M(k, d) & =\sum_{d \mid n} \frac{1}{d} \sum_{c \mid d} \mu(c) k^{d / c} \\
& =\frac{1}{n} \sum_{d \mid n} \sum_{c \mid d} \frac{n}{d} \mu(d / c) k^{c} \\
& =\frac{1}{n} \sum_{c \mid n} \sum_{b \mid \frac{n}{c}} \frac{n}{d} \mu(d / c) k^{c} \\
& =\frac{1}{n} \sum_{c \mid n} k^{c} \sum_{b \mid \frac{n}{c}} \mu(b)\left(\frac{n}{c}\right)\left(\frac{1}{b}\right) \\
& =\frac{1}{n} \sum_{d \mid n} \phi(d) k^{n / d}
\end{aligned}
$$

The last line follows since $\sum_{b \mid \frac{n}{c}} \mu(b)\left(\frac{n}{c}\right)\left(\frac{1}{b}\right)=\phi(n / c)$, where $\phi$ is the Euler's totient function.

For our calculation, $k=2$ since we are only concerned about words with 2 letters or necklaces with 2-coloring. We will denote this Moreau's formula by

$$
M(n)=\frac{1}{n} \sum_{d \mid n} \mu(d) 2^{n / d}
$$

So we have, $\sum_{d \mid n} M(d)=\frac{1}{n} \sum_{d \mid n} \phi(d) 2^{n / d}$.

Proof of lemma 3.4. Note that $C_{n}$ acts on a point of $\mathcal{Z}_{\mathcal{K}}\left(D^{1}, S^{0}\right)$ by cyclically permuting the coordinates. So $C_{n}$ acts freely on all but finitely many points. The coordinate of those points can be only 0 or 1 . Each point in the branch set can be considered a primitive necklace of length $d$ where $d \mid n$. Clearly, there are $M(d)$ points in the branch set which has isotropy group 
$C_{n / d}$. So the summation in the Riemann-Hurwitz formula becomes

$$
\sum_{x \in S}\left(|G|-\frac{|G|}{n_{x}}\right)=\sum_{d \mid n} M(d)\left(n-\frac{n}{n / d}\right)
$$

Now using the Riemann-Hurwitz formula,

$$
\begin{aligned}
& \chi\left(\mathcal{Z}_{\mathcal{K}}\left(D^{1}, S^{0}\right)\right)=n \cdot \chi(X / G)-\sum_{d \mid n} M(d)\left(n-\frac{n}{n / d}\right) \\
& \Longrightarrow(4-n) 2^{n-2}=n \cdot \chi(X / G)-\sum_{d \mid n} n M(d)+\sum_{d \mid n} d M(d) \\
& \Longrightarrow 2^{n}-n 2^{n-2}=n \cdot \chi(X / G)-n \sum_{d \mid n} M(d)+2^{n} \\
& \Longrightarrow \chi\left(\mathcal{Z}_{\mathcal{K}}\left(D^{1}, S^{0}\right) / C_{n}\right)=\sum_{d \mid n} M(d)-2^{n-2} \\
& \Longrightarrow \chi\left(\mathcal{Z}_{\mathcal{K}}\left(D^{1}, S^{0}\right) / C_{n}\right)=\frac{1}{n} \sum_{d \mid n} \phi(d) 2^{n / d}-2^{n-2} \\
& \Longrightarrow g\left(\mathcal{Z}_{\mathcal{K}}\left(D^{1}, S^{0}\right) / C_{n}\right)=1+2^{n-3}-\frac{1}{2 n} \sum_{d \mid n} \phi(d) 2^{n / d}
\end{aligned}
$$

So the quotient space $Z_{K_{n}}\left(D_{1}, S^{0}\right) / C_{n}$ has genus equal to

$$
1+2^{n-3}-\frac{1}{2} \text { (\# of n-length necklace with 2-coloring) }
$$

Example 3.10. For $n=6, Z_{K_{n}}\left(D^{1}, S^{0}\right)$ is a surface with genus $1+(6-$ 4) $2^{6-3}=17$. Under the above formula, the genus of the quotient space $Z_{K_{n}}\left(D^{1}, S^{0}\right) / C_{n}$ is

$$
1+2^{3}-\frac{1}{2} \text { (\# of 6-length necklace with 2-coloring) }
$$

The number of 6 length necklace with 2-coloring is exactly

$$
\frac{1}{6} \sum_{d \mid 6} \phi(d) 2^{6 / d}=\frac{1}{6}\left(1.2^{6}+1.2^{3}+2.2^{2}+2.2\right)=14
$$

So $Z_{K_{n}}\left(D^{1}, S^{0}\right) / C_{n}$ has genus $9-14 / 2=2$. 


\subsection{An upper bound for genus of quotient graph $Q_{n} / C_{n}$}

From the above discussion, we have proved the following lemma.

Lemma 3.11. The genus of the quotient graph, $Q_{n} / C_{n}$ has an upper bound:

$$
\gamma\left(Q_{n} / C_{n}\right) \leq 1+2^{n-3}-\frac{1}{2 n} \sum_{d \mid n} \phi(d) 2^{n / d}
$$

Remark 3.12. It can be showed that theorem 3.3 is also true for a subgroup $C_{m} \subset C_{n}$ where $m \mid n$. In this paper we are not using that result, but I will add a proof of this fact in my $\mathrm{PhD}$ thesis.

\section{Acknowledgements}

The author is thankful to Professor Frederick Cohen for his insightful discussion on the real moment-angle complex and related topics. Also many thanks to the reviewer whose careful reading and suggestions have improved the paper.

\section{References}

[1] F. Harary, J. P. Hayes, H.-J. Wu, A survey of the theory of hypercube graphs, Comput. Math. Appl. 15 (1988) 277-289.

[2] G. Von Ringel, Über drei kombinatorische probleme amn-dimensionalen würfel und würfelgitter, Abhandlungen aus dem Mathematischen Seminar der Universität Hamburg 20 (1955) 10-19.

[3] L. W. Beineke, F. Harary, The genus of the $n$-cube, Canad. J. Math. 17 (1965) 494-496.

[4] V. M. Buchstaber, T. E. Panov, Toric topology, volume 204 of Mathematical Surveys and Monographs, American Mathematical Society, Providence, RI, 2015.

[5] G. Denham, A. I. Suciu, Moment-angle complexes, monomial ideals and Massey products, Pure Appl. Math. Q. 3 (2007) 25-60.

[6] A. Bahri, M. Bendersky, F. R. Cohen, S. Gitler, The polyhedral product functor: a method of decomposition for moment-angle complexes, arrangements and related spaces, Adv. Math. 225 (2010) 1634-1668. 
[7] F. Harary, Graph theory, Addison-Wesley Publishing Co., Reading, Mass.-Menlo Park, Calif.-London, 1969.

[8] J. L. Gross, T. W. Tucker, Topological graph theory, Wiley-Interscience Series in Discrete Mathematics and Optimization, John Wiley \& Sons, Inc., New York, 1987. A Wiley-Interscience Publication.

[9] D. B. West, Introduction to graph theory, Prentice Hall, Inc., Upper Saddle River, NJ, 1996.

[10] A. Perez, Determining the genus of a graph, HC Mathematics Review 1 (2007) 4-13.

[11] R. A. Duke, The genus, regional number, and Betti number of a graph, Canad. J. Math. 18 (1966) 817-822.

[12] L. W. Beineke, F. Harary, Inequalities involving the genus of a graph and its thicknesses, Proc. Glasgow Math. Assoc. 7 (1965) 19-21 (1965).

[13] H. S. M. Coxeter, Regular Skew Polyhedra in Three and Four Dimension, and their Topological Analogues, Proc. London Math. Soc. (2) 43 (1937) 33-62.

[14] A. Al-Raisi, Equivariance, module structure, branched covers, strickland maps and cohomology related to the polyhedral product functor, Ph.D. thesis, University of Rochester NY, 2014.

[15] V. Wm. Pitt Turner, Riemann, Hurwitz, and Branched Covering Spaces, Master's thesis, University of Minnesota, Minneapolis MN 55455, 2011.

[16] C. Moreau, Sur les permutations circulaires distinctes, Nouvelles annales de mathématiques: journal des candidats aux écoles polytechnique et normale 11 (1872) 309-314. 